\title{
Illumination Invariant Feature Extraction for Multispectral Palmprint Verification
}

\author{
Venkateswaran $\mathrm{N}$. \\ Department of Electronics and \\ Communication Engineering, \\ SSN College of Engineering, \\ Chennai -603110
}

\author{
Saranraj S. \\ Department of Electronics and \\ Communication Engineering, \\ MNM Jain College of \\ Engineering, \\ Chennai -603110
}

\author{
Sudharsan S. \\ Department of Electronics and \\ Communication Engineering, \\ SSN College of Engineering, \\ Chennai - 603110
}

\begin{abstract}
The aim of biometrics is to identify humans from their personal traits more efficiently using devices, algorithms and procedures for applications that require security and authentication. Multispectral image analysis has gained importance due to its potential for accurate and faster recognition performance. In this paper, Multispectral palmprint biometric system is proposed which uses the fusion of both MS and visible image to acquire more discriminative palm print information. The proposed system collects palm print images in visible and NIR bands. PCA based Fusion algorithm has been used to obtain more informative palmprint. First, Region of Interest (ROI) is extracted from the acquired palm print images. Then, features are extracted using phase congruency, histogram of gradient, Gabor filter and adaptive thresholding based algorithms. Simple distortion based measures are used for recognition. The proposed system is tested on a palmprint data collected using 080GE multispectral camera. Simulation results show high recognition performance using Gabor features obtained by fusion of visible and NIR palm print image.
\end{abstract}

\section{Keywords}

Biometrics, Multispectral image, Region of interest (ROI), Phase Congruency, HoG, Adaptive thresholding, Gabor filter

\section{INTRODUCTION}

Biometrics is the study of measurable biological characteristics. It mainly based on analyzing certain human body characteristics, such as fingerprint, palmprint, retina, iris, face, voice patterns etc.,. Biometrics based identification/verification is playing an important role in modern personal identification systems. Such systems are increasingly found in public and consumer security systems, point of scale (POS) and consumer electronics. There has been a growing demand for personal identification systems that is more convenient and secured than traditional methods such as passwords, ID (Identity) cards etc. The emergence of personal mobile devices has increased the application domain of biometric systems. More and more biometric features are used in commercial systems for surveillance, fraud detection etc.

Palm print based biometric systems have been researched over a decade. The complex structure of palm print mainly contains three major flexions, the principal lines, secondary lines (wrinkles) and ridges. These complex structures are different for even identical twins and thus are very useful in personal identification. Multispectral biometric data contains information at different wavelengths of the same object. A multispectral image can avoid a metamerism (similar in structures but not all the structures are entirely alike) problem of an RGB image.

Robert K Rowe (2007) developed a Multispectral whole-hand biometric authentication system by extracting finger print features and palm print features. 2-D Gaussian function with different orientation is used to extract features. A dissimilarity score is calculated by averaging the normalized Hamming distance of the feature vectors. It is shown that features obtained from the fingerprint and palm print images are combined to improve the recognition performance. Jong Hyun Park (2007) used multispectral infrared iris image and showed that fusion of images at different wavelengths to form a single grey scale iris image using gradient based image fusion method. In gradient based fusion method, first order gradient information is calculated for iris images obtained at different wavelengths. Then information is maximized by integrating into a single grey scale image. After the fusion step, the general iris recognition step is used for authentication. The fused result of real image and fake images are compared using Hamming distance. For fake iris, the hamming distance is generally very high.

Ying Hao (2007) developed several multispectral decomposition methods for multispectral image fusion such as gradient pyramid, morphological pyramid and shift invariant DWT. They proposed bilateral filtering and pixel level fusion helps in preserving the important features like principal lines, wrinkle lines, blood vessels. David Zhang (2010) developed a prototype for multispectral palm print system. ROI is extracted by taking the gaps between the fingers as reference points. The ROI of the palm image is split into nonoverlapping blocks and histogram of orientation gradient for individual block is extracted. It uses Block dominant orientation code (BDOC) as a rough feature and the Block based histogram of orientation gradient (BDHOG) as fine feature. The hierarchical classifier is developed by calculating the Hamming distance as a matching metric for classification of rough and fine features. In addition, a normalized correlation coefficient (NCC) is also calculated to measure the similarity. Based on the hamming distance and NCC, the final scores of the images are determined. 


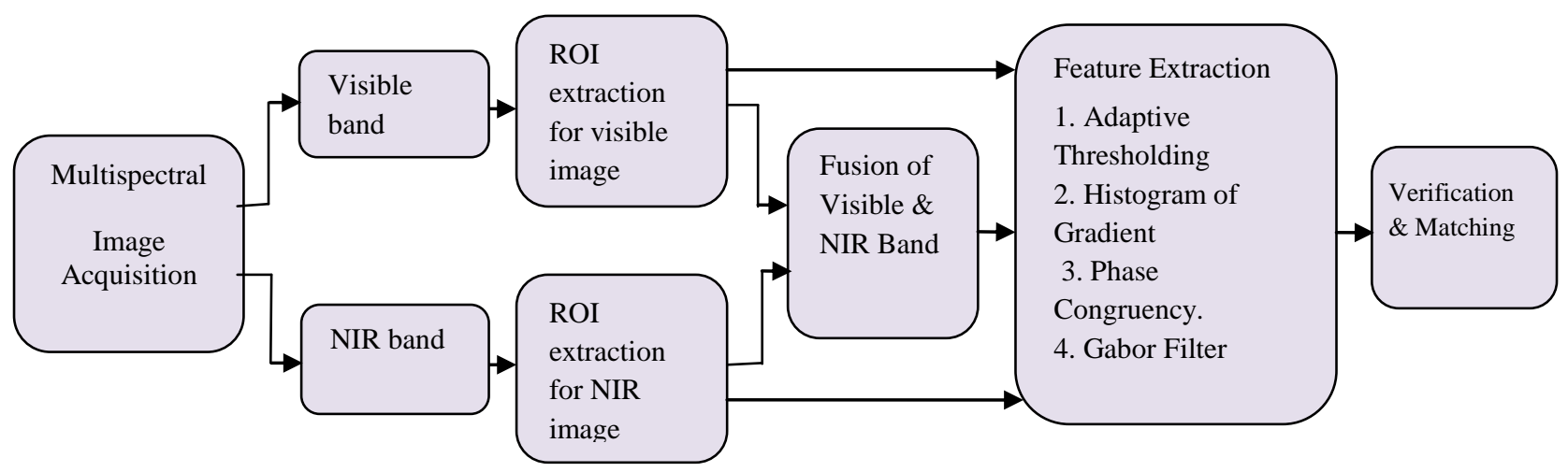

Fig 1: Palm print biometric recognition system with multispectral image fusion

ZhenhuaGuo (2012) presented the method to identify the number of feature bands for multispectral palm image. This system includes components such as liquid crystal tunable filter (LCTF), charge coupled device (CCD) and illumination source. ROI is extracted from the hyperspectral palm image and a clustering based method is presented to identify the number of feature bands from the database. Initially the similarity of the two images is calculated by using complex wavelet structural similarity. It is calculated by computing complex wavelet transform of those images which is invariant to lumination and contrast using theeigen values of the similarity matrix of the palm image. Then the image features are extracted by using K-means clustering. It is found that the most of the discriminative information is present in the three spectral bands. Christoph Busch (2014) proposed a method for multispectral palmprint recognition using fusion technique. Wavelet coefficients of Multispectral of palmprint image are combined either by subband selection or subband weighting by the vlues of mutual information function. Log Gabor Transform is applied to the palm print image for feature extraction. Across different bands and also robust method is enough for person verification by palm print recognition.

Similar to the above of line of research, our contribution to this paper is to evaluate various feature extraction methods in representing lines and wrinkles that is invariant to illumination and contrast. In addition, we are able to show that the performance of palm print biometric verification improves using fusion strategy on multispectral palmprints.

The remaining sections of this paper are organized as follows. Section II briefly introduces the proposed palm print recognition system. Section III presents experimental results Section IV provides comparative results of palmprint features, and Section V summarizes this paper.

\section{MULTISPECTRAL PALMPRINT RECOGNITION SYSTEM}

In general, at the input, biometric system consists of scanning device or a reader. The scanned information is converted to digital data with a help of software. A database is created for comparison which stores the data. The flow diagram of proposed multispectral palm print recognition system that consists of four parts is shown in Fig.1. The first part extracts region of interest consisting of important details from both visible and IR based images of palm print. A novel segmentation algorithm has been proposed that precisely represent differentiating features between individuals. The second part of the framework is fusion ROI extraction of multispectral images to obtain a more comprehensive palm print image. Feature extraction is the third part of our proposed framework. Here four different methods are employed independently for extraction of features that are invariant to illumination. The four algorithms are evaluated for the precise representation of the palm print feature. Finally, a matching function was employed for authentication/verification of individuals. The proposed approach also considers variation in illumination of multispectral image acquisition in the image acquisition stage.

\subsection{Image Acquisition and Database}

The Multispectral palmprint image acquired using AD-080GE multispectral camera. The camera setup consists of lens arrangement of capture multispectral information. The system captures visible images in the spectral range (400-700nm) and Near Infrared Red images in the spectral range (800-1000nm) images which are used for the further processing. In visible range, skin surface components of the palmprint images are captured. In NIR image, vein information of the palmprint image is obtained. The acquired visible and NIR image is pre-processed in order to remove the noise and adjust the dynamic range. The multispectral camera used to capture the multispectral palm print images is shown in Fig.3. In general, for palmprint biometric verification, the use of entire palmprint information by including region of non interest may degrade the accuracy of verification. Therefore, in this system, before feature extraction the Region of Interest is extracted.

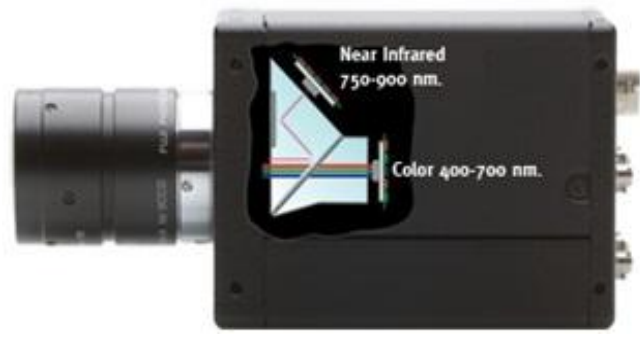

Fig 2: Multispectral camera for image acquisition

\subsection{ROI Extraction}

The acquired image is subjected to ROI segmentation with the help of certain co-ordinates points using the vertical intensity profile of the palm image. The vertical profile is calculated using vertical profile for $1,2,3 \ldots \mathrm{n}$ where $\mathrm{X}(\mathrm{i}, \mathrm{j})$ be the input image and $(m, n)$ is the size of rows and columns. 


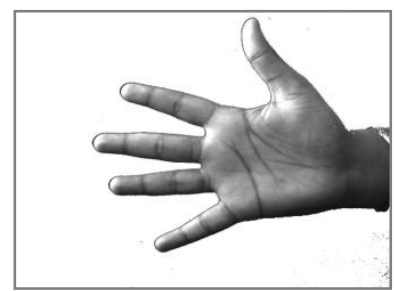

(a)

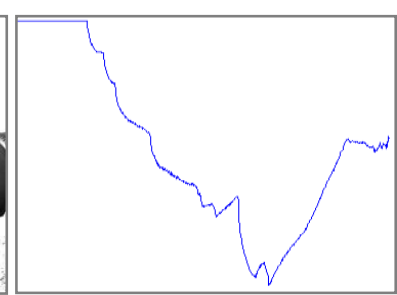

(b)
Fig 3: Palm Print Image and the Vertical Intensity profile

The algorithm for segmenting ROI from the acquired data is as follows

Step1: Two extreme points $\mathrm{P} 1$ and $\mathrm{P} 2$ are marked in the palm image with respect to the vertical profile as shown in Figure 3. Point P1 is the first pixel with a value is different from 255 when scanned from left to right and Point P2 is the Midpoint from whose value is different from 255 pixels in the column of Y.

Step2: The point P3 is marked as the midpoint of P1 \& P2.

Step3: Similarly points P4, P5, P6, P7 and P8 are the midpoints of P2 \& P3, P3 \& P4, P2 \& P4, P3 \& P5 and P4 \& P6 respectively.

Step4: The points P7and P8 are taken as reference points and a square is completed in the palm print image.

Step5: Let ' $d$ 'be the distance between the points P7 and P8 and the corner co-ordinate points of the square is marked by moving the points P7 \& P8 upwards and downwards by the pixel distance of $\mathrm{d} / 2$.

Step6: Finally, the ROI is cropped by using the corner coordinate points as shown in Fig.4

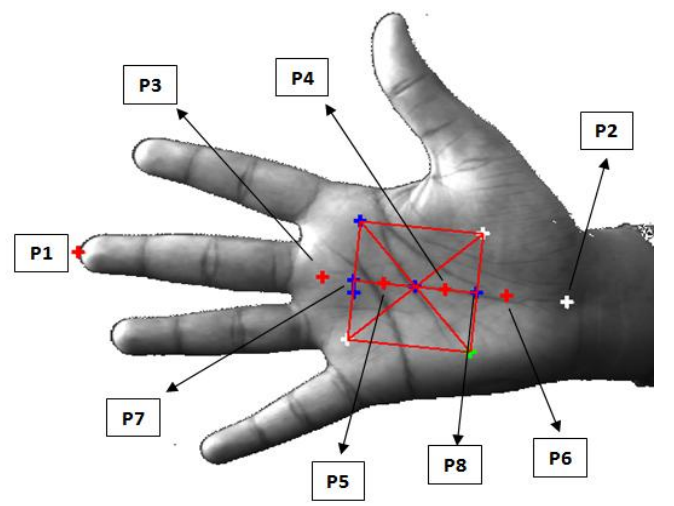

Fig 4: Region of Interest Extraction of Input Palmprint

A multi-spectral image is a collection of several monochrome images of the same scene, each of them taken with different sensors. Palm print ROI extraction obtained by using the above algorithm is shown in Fig.5. It shows Palmprint ROI images are extracted for visible band, NIR band and fused version of both visible and NIR band. Visible band is a RGB color image, consisting of a red, a green and a blue image, each of them taken with a sensor sensitive to a different wavelength. It ranges from 400-700nm in the electromagnetic spectrum. NIR band is an invisible radiant energy in the electromagnetic spectrum with longer wavelengths than the visible band. In this multispectral camera, NIR band is captured at a different wavelength which ranges from 750$900 \mathrm{~nm}$.

\subsection{Visible and NIR Palmprint Image Fusion using PCA}

The goal is to obtain a high fidelity image from the set of captured images. Principal Component Analysis is used to extract relevant information from the two band of palmprint images. PCA is a statistical technique that has number of application in fields such as face recognition and image compression is adopted for visible and NIR palmprint image fusion. PCA analyses the covariance of the data and captures principal components using Eigen analysis. Given the Visible palmprint Image $\mathrm{I}_{\mathrm{V}}(\mathrm{x}, \mathrm{y})$ and Near Infrared palmprint Image $\mathrm{I}_{\mathrm{NIR}}(\mathrm{x}, \mathrm{y})$.The normalized components $\mathrm{PC} 1$ and PC2 respectively are computed using PCA. The fused image $\mathrm{I}_{\mathrm{F}}(\mathrm{x}, \mathrm{y})$ is given by

$$
I_{F}=P_{c 1} I_{V}+P_{c 2} I_{N I R}
$$

The important steps followed in PCA based multispectral fusion of palmprint images are:

Step 1: The visible and near infrared are arranged in a data matrix (P) consisting of two column vectors, where each column vector represents palmprint images.

Step 2: The empirical mean along each column is computed and subtracted from the respective column of the data matrix (P).

Step 3: The covariance matrix $\mathrm{P}$ is computed using $\mathrm{C}=\mathrm{P}^{\mathrm{T}} \mathrm{P}$.

Step 4: Then, the Eigen values (D) and Eigen vector (E) of the data matrix $(\mathrm{P})$ is computed and arranged in decreasing order of its Eigen value. Both (D) and (E) are resized 2 x 2 .

Step 5: The normalized principal components are calculated using,

$P_{c 1}=\frac{E(1)}{\sum E} P_{c 2}=\frac{E(2)}{\sum E}$

Such that $\mathrm{P}_{\mathrm{c} 1}+\mathrm{P}_{\mathrm{c} 2}=1$

Step 6: The two palmprint images are fused using a weight averaging fusion rule based on principal components given in equation 2 .

\subsection{Feature Extraction}

\subsubsection{Adaptive Thresholding}

The palm print is a grayscale image. A Palmprint recognition system has to be robust against any variations in illumination of the input palm print. Thresholding techniques are generally employed to classify input pixels as either "black" or "white". Adaptive thresholding is a better solution compared to "simple" thresholding since; it takes into account spatial variations of illumination in the multispectral palm image. Adaptive thresholding using integral of the input image employed here is an extension of Wellner's method. An integral image has been applied to texture mapping, face detection, and stereo correspondence. The integral image $\mathrm{I}(\mathrm{x}, \mathrm{y})$ is computed at each pixel location $(\mathrm{x}, \mathrm{y})$ by taking into account the border cases, as the sum of all $\mathrm{G}(\mathrm{x}, \mathrm{y})$ terms to the left and above the pixel (x,y) given by,

$$
I(x, y)=G(x, y)+I(x-1, y)+I(x, y-1)-I(x-1, y-1)
$$


Adaptive thresholding is performed as a two step process on the palmprint images. The integral image is calculated in the first step and in the second step, the average of a $s x s$ window of pixels centered on each pixel is calculated. Finally, if the value of the current pixel is $t$ percent less than this average then it are set to black, otherwise it is set to white. It gives a better average for comparison since it considers neighboring pixels on all sides.

\subsubsection{Histogram of gradient}

A typical Palm pint contains varied and complex patterns that are unique to every individual. HOG is a spatial descriptor which is sensitive to gradient orientations and it is more suitable to extract features for palm print recognition. In the spatial domain, the orientation features that resembles like edge map, yields texture information based on the variations on the intensity values over the palm print. Let $\mathrm{G}$ be the ROI extracted palm print. By filtering $\mathrm{G}$ with $\mathrm{I}=\left[\begin{array}{lll}-1 & 0 & 1\end{array}\right]$ and we obtain two images $G_{x}$ and $G_{y}$. These are known as

horizontal and vertical gradient maps of the palm print image. For every pixel in $\mathrm{G}$, the magnitude $\operatorname{mag}(G(i, j))$ and gradient orientation angle ang $(G(i, j))$ are calculated as,

$$
\begin{aligned}
& G_{x}(i, j)=I * G \\
& \text { (4) }_{y}(i, j)=I^{T} * G
\end{aligned}
$$

$$
\operatorname{mag}(i, j)=\sqrt{{G_{x}}^{2}+G_{y}^{2}} \operatorname{ang}(i, j)=\tan ^{-1}\left(G_{y} / G_{x}\right)(6)
$$

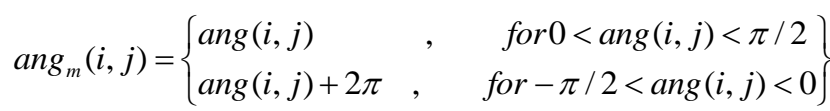

where '*' is a convolution operator. The ang $(i, j)$ that is changing in $(-\pi d 2, \pi d 2)$ is transformed to $(0,2 \pi)$ for easy manipulations as,

The Orientation of the gradient map is empirically calculated as

$\operatorname{ang}_{f}(i, j)=\left\{\begin{array}{l}\operatorname{ang}_{m}(i, j) \operatorname{for}_{x}(i, j)>=0 \\ \operatorname{ang}_{m}(i, j)+\pi \operatorname{for}_{x}(i, j)<0 \& \operatorname{ang}_{m}(i, j)<\pi / 2 \\ \operatorname{ang}_{m}(i, j)-\pi \operatorname{for}_{x}(i, j)<0 \& \operatorname{ang}_{m}(i, j)>3 \pi / 2\end{array}\right\}$

$f_{k}=\sum \operatorname{mag}(i, j)$ if $(k \pi / 4)<\operatorname{ang}_{f}(i, j)<(k+1) \pi / 4$ Where, $\operatorname{ang}_{\mathrm{f}}$ gives some important features and $\operatorname{ang}_{\mathrm{m}}$ is an intermediate variable for representing $\operatorname{ang}_{\mathrm{f}}$. Orientation histogram of gradient can be extracted from the orientation maps of pixel point by covering $360^{\circ}$ range of orientation. Histogram is weighted by $\operatorname{mag}(i, j)$ which is added to the histogram and Orientation histogram of gradient is expressed as follows,
Hence the Fig.6 shows the extracted features by using Histogram of gradient at different bands such as visible and NIR bands. However, these features are highly sensitive to variations in illumination; blurring and scaling. The performance is also dependent on the choice of the threshold.

\subsubsection{Phase Congruency}

A typical palmprint contains number of step discontinuities in intensity. Edges and corners are detected by phase congruency method is robust and invariant to contrast and scale. Phase congruency is the multi-orientation and multi-scale approach and it emphasis phase rather than magnitude information. It uses number of 2D log-Gabor filters with different scales. This feature map is based on the Peter Kovesi phase congruency model. Then a conversation to binary form must be performed. We choose a global threshold for this purpose. This global threshold has been obtained from Otsu global thresholding method. The phase congruency map is determined using kovesi's model. The local frequency information is calculated by convolving the palmprint image $\mathrm{G}(\mathrm{x}, \mathrm{y})$ with quadratture pairs of $\log$ Gabor wavelets.

$$
\left[e_{\rho}(x, y), o_{\rho}(x, y)\right]=\left[G(x, y) * M_{\rho}^{e}, G(x, y) * M_{\rho}^{o}(11)\right.
$$

Where $M_{\rho}^{e}$ and $M_{\rho}^{o}$ denotes the even and odd symmetric log Gabor wavelet at a scale $\rho$. The signal $e_{\rho}(x, y)$ and $\mathrm{O}_{\rho}(\mathrm{x}, \mathrm{y})$ are the even symmetric filter output and odd symmetric filter output respectively at different scales that encode phase and amplitude. Then the local phase $\varphi_{\rho}(\mathrm{x}, \mathrm{y})$ and local amplitude $\mathrm{A}_{\rho}(\mathrm{x}, \mathrm{y})$ is computed by using the wavelet responses as,

$$
\begin{aligned}
& \psi_{\rho}(x, y)=\arctan 2\left(o_{\rho}(x, y), e_{\rho}(x, y)\right) \\
& \mathrm{A}_{\rho}(\mathrm{x}, \mathrm{y})=\sqrt{\left.\mathrm{e}_{\rho}(\mathrm{x}, \mathrm{y})^{2}+\mathrm{o}_{\rho}(\mathrm{x}, \mathrm{y})^{(12}\right)}
\end{aligned}
$$

The phase congruency is the ratio given by

$$
P C(G(x, y))=\frac{|E(G(x, y))|}{\sum_{\rho} A_{\rho} G(x, y)}, \quad \text { with } 0 \leq P C \leq(G(x, y)) \leq 1
$$

$$
\operatorname{PC}(x, y)=\frac{\sum_{\rho} W(x, y)\left|A_{\rho}(x, y) \Delta \varphi_{\rho}(x, y)\right|}{\sum_{\rho} A_{\rho}(x, y)+\varepsilon} \text { Phase congruency lies }
$$

between no congruency (0) to perfect congruency (1). Phase congruency is a normalized quantity and provides image features that are invariant to contrast and scale. However, Phase congruency is sensitive to noise. Also, images have frequency dependent amplitude spectrum that varies as $1 / f$. If the noise spectrum is assumed to be to be flat, then the response of smallest log - Gabor wavelet filter is mostly energy from noise, because of its larger bandwidth. This reduces the local information from the image. In order to improve the localization, a modified phase congruency map is defined as 
Where $\varepsilon$ is small constant to avoid division by zero and $\mathrm{W}(\mathrm{x}, \mathrm{y})$ is the frequency spread weighting function defined as

$$
\begin{array}{r}
\mathrm{W}(\mathrm{x}, \mathrm{y})=\frac{1}{1+\mathrm{e}^{\gamma(\mathrm{c}-\mathrm{s}(\mathrm{x}, \mathrm{y}))}} \\
s(x, y)=\frac{1}{N}\left(\frac{\sum_{\rho} A_{\rho}(x, y)}{A_{\max }(x, y)}\right)
\end{array}
$$

and

Where $s(x)$ is the fractional measure of spread; $N$ represents the total number of filter scales; $A_{\max }$ denotes the amplitude of the filter pair with maximum response; $C$ is the cut-off value and $\gamma$ is the gain factor. Where, $\Delta \phi_{\rho}$ is phase deviation function is given by,

$$
\Delta \varphi_{\rho}=\cos \left(\varphi_{\rho}(\mathrm{x}, \mathrm{y})-\bar{\varphi}(\mathrm{x}, \mathrm{y})\right)-\mid \sin \left(\varphi_{\rho}(\mathrm{x}, \mathrm{y})-\bar{\varphi}(\mathrm{x}, \mathrm{y}) \mid\right.
$$

Where $\varphi_{\rho}(\mathrm{x}, \mathrm{y})-\bar{\varphi}(\mathrm{x}, \mathrm{y})$ the phase deviation at scale $\rho$, $\bar{\varphi}(\mathrm{x}, \mathrm{y})$ denotes the mean phase angle.

\subsubsection{Orientation Features using Gabor Filter}

Gabor filter has been used to extract texture features to capture the contrast information. For feature extraction of multispectral palm image, Gabor filter is more suitable as the palm print mostly contains line information that are darker than the background. Gabor filter can be applied along different directions to the palm print images to obtain orientation feature. The real Gabor filter kernel at angle $\theta=\pi / 2$ can defined as

$$
g(x, y)=\frac{1}{2 \pi \sigma_{x} \sigma_{y}} \exp \left(-\left(\frac{x^{\prime 2}+y^{\prime 2}}{2 \sigma^{2}}\right) \cos \left(2 \pi f_{0} x\right)\right)
$$

where $\sigma_{\mathrm{x}}, \sigma_{\mathrm{y}}$ are standard deviation values along $x$ and $y$ directions and $f_{0}$ is the frequency of modulating sinusoid. Gabor filter kernel for other orientations can be obtained from co-ordinate transformation,

$$
x^{\prime}=x \cos \theta+y \sin \theta
$$

$$
y^{\prime}=-x \sin \theta+y \cos \theta
$$

Where $-\pi / 2<\theta<\pi / 2$

The Gabor filter is applied to all the three input multispectral palm print images. Features are extracted at different orientations and the orientation with complete information is used for feature extraction.

\subsection{Matching and Identification}

For the extracted features described in section III, the matching has been performed with two matching scores namely Distance reciprocal distortion (DRD) and F-measure to arrive at the final decision.

\subsubsection{Distance-Reciprocal Distortion}

Distance-Reciprocal Distortion measures the distortion of the extracted feature map by comparing with the feature map of database palm print images. It computes a weighted matrix with each of its weights determined by the reciprocal of a distance measured from the center element. Assuming the weight matrix $W m$ is of size $m \times m$, where $\quad m=2 n+1$ and $n \varepsilon N$. The center element of this matrix is at $\left(i_{c}, j_{c},\right)$ where $i_{c}=j_{c}=(m+1) / 2$.

$W_{m}(i, j)=\left\{\begin{array}{cc}0 & \text { for } i=i_{c}, j=j_{c} \\ \frac{1}{\sqrt{\left(i-i_{c}\right)^{2}+\left(j-j_{c}\right)^{2}}}, & \text { for otherwise }\end{array}\right\}$

This weighted matrix is used to obtain normalized weighted matrix is given by,

$$
W_{N m}(i, j)=\frac{W_{m}(i, j)}{\sum_{i=1}^{m} \sum_{j=1}^{n} W_{m}(i, j)}
$$

For the pixels in the feature map that is different from the considered matching palmprint, a distance reciprocal distortion is computed. The DRD measured for a flipped (form black to white or form white to black) pixel is given by

$$
D R D_{k}=\sum_{i, j}\left[D_{k}(i, j) \quad X \quad W_{N m}(i, j)\right]
$$

Where the $(i, j)$ th element of the difference matrix is given by,

$$
D_{k}(i, j)=\left|B_{k}(i, j)-g(x, y)_{k}\right|
$$

Thus, DRD at any flipped pixel equals to the weighted sum of the pixels in the blocks $B_{k}(i, j)$ of the original image feature map that differ from the flipped pixel in the $g(x, y)_{k}$ database palm print image. Total distortion is calculated by adding all the distortions of from each flipped pixels and normalized over non uniform pixel blocks.

\subsubsection{F-Measure}

The F- measure can be interpreted as a weighted average of the sensitivity (Precision) and specificity (Recall). FMeasure is a measure of accuracy. Precision is also called as positive predictive value which is the fraction of retrieved instances that are relevant, whereas recall is the fraction of relevant instances that are retrieved. The precision and recall parameters are measured as,

Sensitivity $($ precision $)=\frac{\text { Number of true positives }}{\text { Number of true positives }+ \text { Number of false positives }}$

Specificity $($ recall $)=\frac{\text { Number of true negatives }}{\text { Number of true negatives }+ \text { Number of false positives }}$ 
The F- measure is the harmonic mean of Sensitivity/Specificity and it determines the test's ability to correctly detect perfect matching.

$$
F=2 \cdot \frac{\text { Precision } \times \text { Recall }}{\text { Precision }+ \text { Recall }}
$$

By using these two parameters, matching scores has been computed for verification of different palm images at different bands with a user input palm print.

\section{SIMULATION RESULTS}

In this section, experimental results for the proposed palmprint recognition method are presented. The palm print belonging to different subjects was captured using the multispectral camera (AD-080GE) shown in Fig.3. The biometric features were extracted using four different techniques namely, phase congruency, Histogram of gradient, Gabor filter orientation features and Adaptive thresholding. The extracted features thus form the palmprint database. The test input is captured with no strict constraints on the environment with respect to distance of the palm, illumination requirements, background, noise etc. and at different time instants. It is therefore highly unlikely that the contrast, brightness and other conditions are same as that of the templates. The test features were also extracted using the four aforementioned techniques.

The simulation has been performed in a workstation with Intel dual core processor $(1.8 \mathrm{GHz})$ with 4GB RAM using Matlab 15 for Windows. The extracted test features were compared against all the templates existing in the database. Fig 5. ((a-e) visible band, (f-j) in NIR bands and (k-o) in the fused images) show the different extracted features for five sample palm print images using adaptive thresholding method. Similarly, Fig.6 shows the extracted features by using Histogram of gradient method at different bands such as visible and NIR bands. The phase congruency map is shown in the Fig.7. Finally, Fig. 8 shows the Features obtained by applying Gabor filter at $90^{\circ}$ orientation.

Four different metrics were used to obtain the matching scores namely Distortion Reciprocal Distortion (DRD) and Fmeasure. The same process was applied to the visible band, NIR band and fused versions of the database samples and test input. Table.1 shows the matching scores computed by comparing visible test input palm against the samples in the visible database palm and similarly with other two sets of images. From the matching scores, it can be inferred that Gabor filter based feature extraction gives the most accurate results when applied to biometric authentication based applications.

Based on the results for the NIR images the following observations can be noticed. Matching scores based only on NIR images are poor among the set of images considered. The visible palm print images have produced considerably better matching scores. Compared to NIR palmprint images, the PCA based fused image has consistently presents high matching scores among the three types of input palmprint images. The high matching scores obtained for Gabor filter based feature extraction indicates that, this technique is more accurate and greater usability for palmprint based biometric applications. Similarly Table.1 also gives the matching score for fused versions of the NIR and Visible images. It is also evident that Gabor orientation features gives the most accurate results when the fused images are available.

\section{CONCLUSION}

In this paper, a multispectral palmprint biometric identification system with no significant constraints on the environment is investigated. In the proposed approach, using features that are less sensitive to illumination invariance such as phase congruency, histogram of gradient, adaptive thresholding and Gabor filter is extracted. The visible and NIR palm print images are fused to obtain new features. The ability of biometric fusion was demonstrated by calculating matching scores and compared with scores obtained using visible and NIR images. Simulation results demonstrate the superior biometric recognition results using Gabor filter orientation features obtained from fusion of visible and NIR image for palm print images.

\section{REFERENCES}

[1] Patrick Flynn, 2008. Handbook on Biometrics. Springer.

[2] David Maltoni, Anil.K.Jai, et.al.,2006. Handbook on Multibiometrics. Inger-Verlag.

[3] Yuan Yan Tang, Yang Lu,et.al. 2015. Hyperspectral Image Classification Based on Three-Dimensional Scattering Wavelet Transform. IEEE Transactions on Geoscience and Remote sensing, vol. 53, 2467-2476.

[4] Ruud Bolle, SharathPankanti,et.al. 2002. Biometrics personal identification in Network society. Springer, Heidelberg, 2002.

[5] Dong Han, ZhenhuaGuo, et.al. 2008 Multispectral Palmprint Recognition Using Wavelet-Based Image Fusion. IEEE Transactions on Biometric computing and Image Processing, 2074-2077.

[6] M. P. Sampat, Z. Wang, S. Gupta, A. C. Bovik, and M. K. Markey 2009. Complex wavelet structural similarity: A new image similarity index. IEEE Trans. Image Process., vol. 18, no. 10, 2385-2401.

[7] D. Zhang,2000. Automated Biometrics-Technologies and Systems. MA: Kluwer.

[8] David Zhang, ZhenhuaGuo, Guangming Lu, et.al. 2010. An Online System Of Multispectral palm print Verification IEEE Transactions on Instrumentation and Measurement, vol. 59, no. 2, 480-490.

[9] Online Multispectral Palmprint Recognition 2012. IEEE Transactions On Information Forensics And Security, vol. 7, No. 3, 1094-1099. 2012

[10] R. Raghavendra n, Christoph Busch, et.al. 2014. Novel Image Fusion Scheme Based On Dependency Measure For Robust Multispectral Palmprint Recognition. International Journal of Advanced Research in pattern recognition, vol. 47, 2205-2221.

[11] Robert K Rowe, UmutUludag, et.al. 2007. A Multispectral Whole-Hand Biometric Authentication System. IEEE Transactions on Biometric Recognition Systems.

[12] Ying Hao, Zhenan Sun, et.al .2010. Comparative Studies On Multispectral Palm Image Fusion For Biometrics. 
National Laboratory of Pattern Recognition, Institute of Automation, CAS.

[13] Simona Crihalmeanu, Arun Ross, et.al., 2012. Multispectral Scleral Patterns for Ocular Biometric Recognition. IEEE Transactions on pattern Recognition Systems, vol. 33, 1860-1869.

[14] Danfeng Hong, Wanquan Liu, et. al. 2015. A Novel Hierarchical Approach For Multispectral Palmprint Recognition. IEEE Transactions on Neuro computing, 511-521.

[15] Rajashree Bhokare, Deepali Sale . 2013 Multispectral Palm Image Fusion: A Critical Review. International Journal of Advanced Research in Computer Engineering \& Technology, 2159-2164.

[16] Ancy.S, G.R.Suresh, et.al.2013. Survey On Multispectral Biometric Images. International Journal of Innovative Research in Computer and Communication Engineering. 1025-1036.

[17] Maurício Ramalho, Sanchit Singh 2011. Secure MultiSpectral Hand Recognition System IEEE Signal Processing Conference, 2269-2273.

[18] D. Zhang, W. Kong, J. You and M. Wong 2003. Online Palm print Identification. IEEE Transactions on Pattern Analysis and Machine Intelligence. 1041- 1050.

[19] T. Connie, A. T. B. Jin, and M. G. K. Ong 2005. Automated palms print recognition system. Image Vis. Comput. 501-505.

[20] D. Hu, G. Feng, and Z. Zhou 2007. Two-dimensional locality preserving projections (2DLPP) with its application to palm print recognition. Pattern Recognit. 339-342,
[21] C. Han, H. Cheng, C. Lin, and K. Fan.2003. Personal authentication using palm-print features. Pattern Recognition. 371-381.

[22] X. Wu, D. Zhang, and K. Wang. 2006. Palm line extraction and matching for personal authentication. IEEE Trans. Syst., Man, Cybern. A, Syst., Humans. 978-987. 2006.

[23] N. Otsu.1979. A threshold selection method from graylevel histograms. IEEE Trans. Syst., Man, Cybern.,Syst. $62-66$.

[24] P. Kovesi.1999.Image features from phase congruency. J. Comput. Vis. Res. 1-26, 1999.

[25] Bradley, D. and Roth, G. 2007. Adaptive thresholding using the integral image. J. Graph. Tools. 13-20

[26] Saranraj, S. Venkateswaran,N. 2014. Enhancement of mobile camera captured document image with phase preservation. Proc. ICCNT. 68-73.

[27] Saranraj.S. Venkateswaran, N. 2015. Efficient Illumination Correction for Camera Captured Image Documents, Adv. in Nat. Appl. Sci., 391-396.

[28] A. Kong, D. Zhang, and M. Kamel,2006 Palmprint identification using feature-level fusion Pattern Recognition,. 478-487.

[29] A. Kong and D. Zhang 2004. Competitive coding scheme for palmprint verification. Int. Conf. Pattern Recog. 520 523.

[30] W. Jia, D.-S. Huang, D. Zhang 2008. Palmprint verification based on robust line orientation code. Pattern Recognition. 1504- 1513.

\section{APPENDIX}

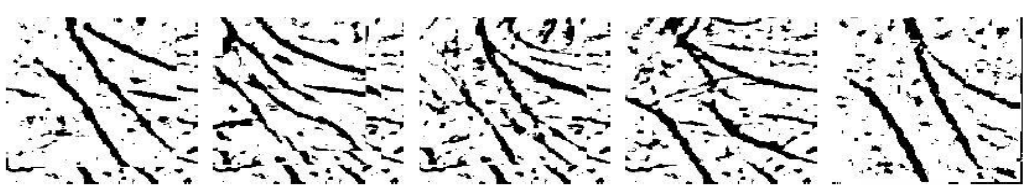

(a)

(b)

(c)

(d)

(e)

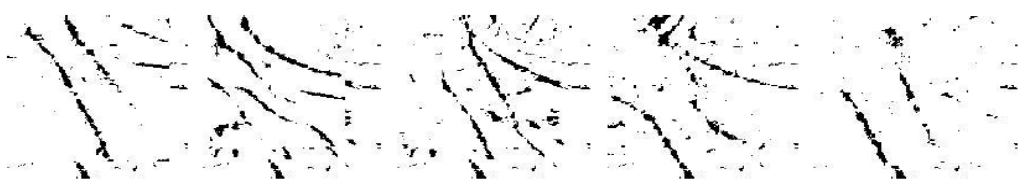

(f)

(g)

(h)

(i)

(j)

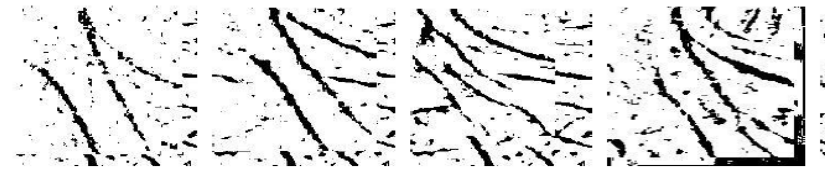

(k)

(I)

(m)

(n)

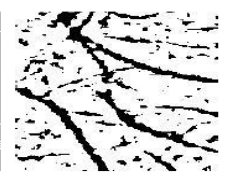

(o)

Fig 5: Extracted feature map from (a-e) visible band, (f-j) NIR bands and (k-o) fused images using adaptive thresholding method 


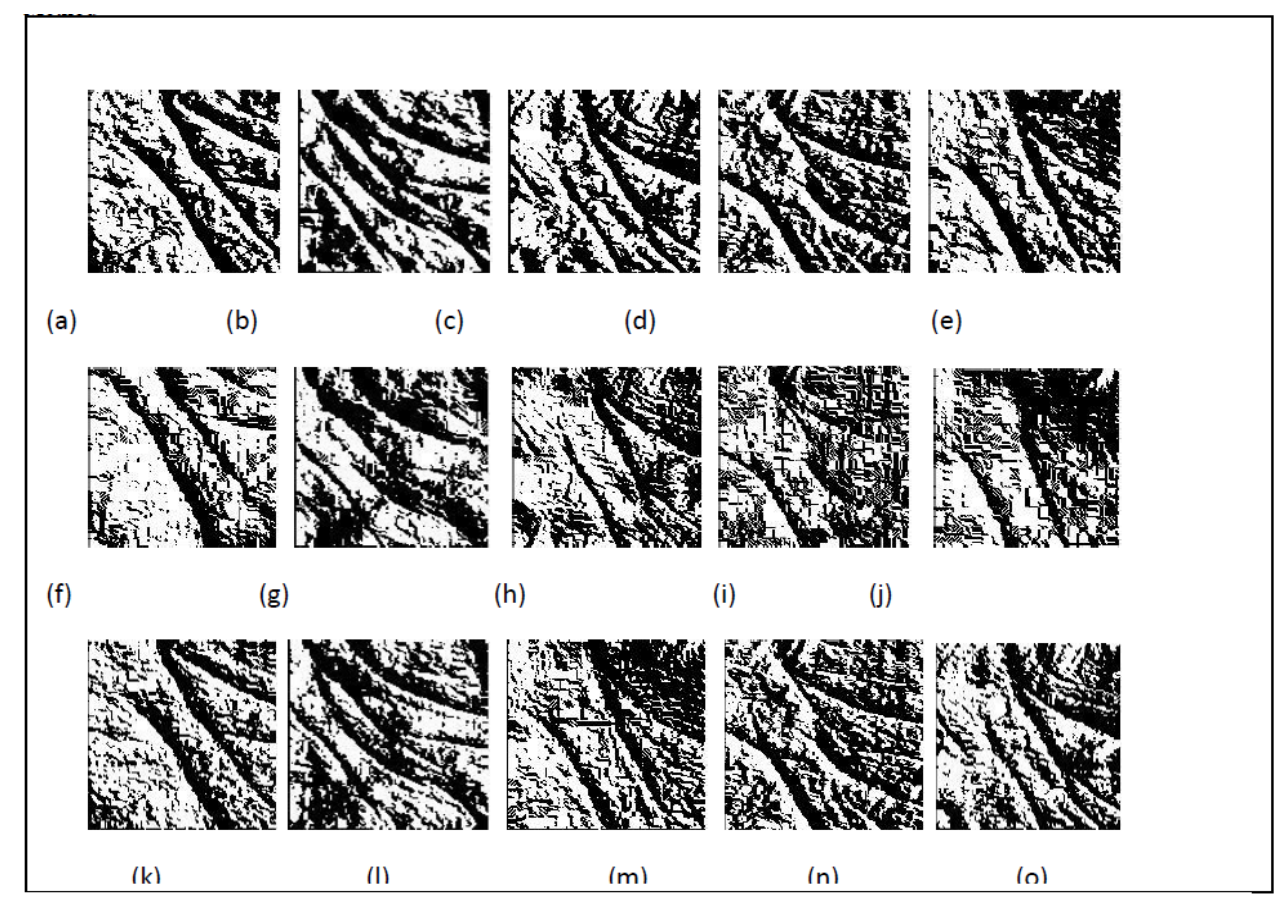

Fig 6: Extracted feature map (a-e) visible band, (f-j) NIR bands and (k-o) fused images using HOG method

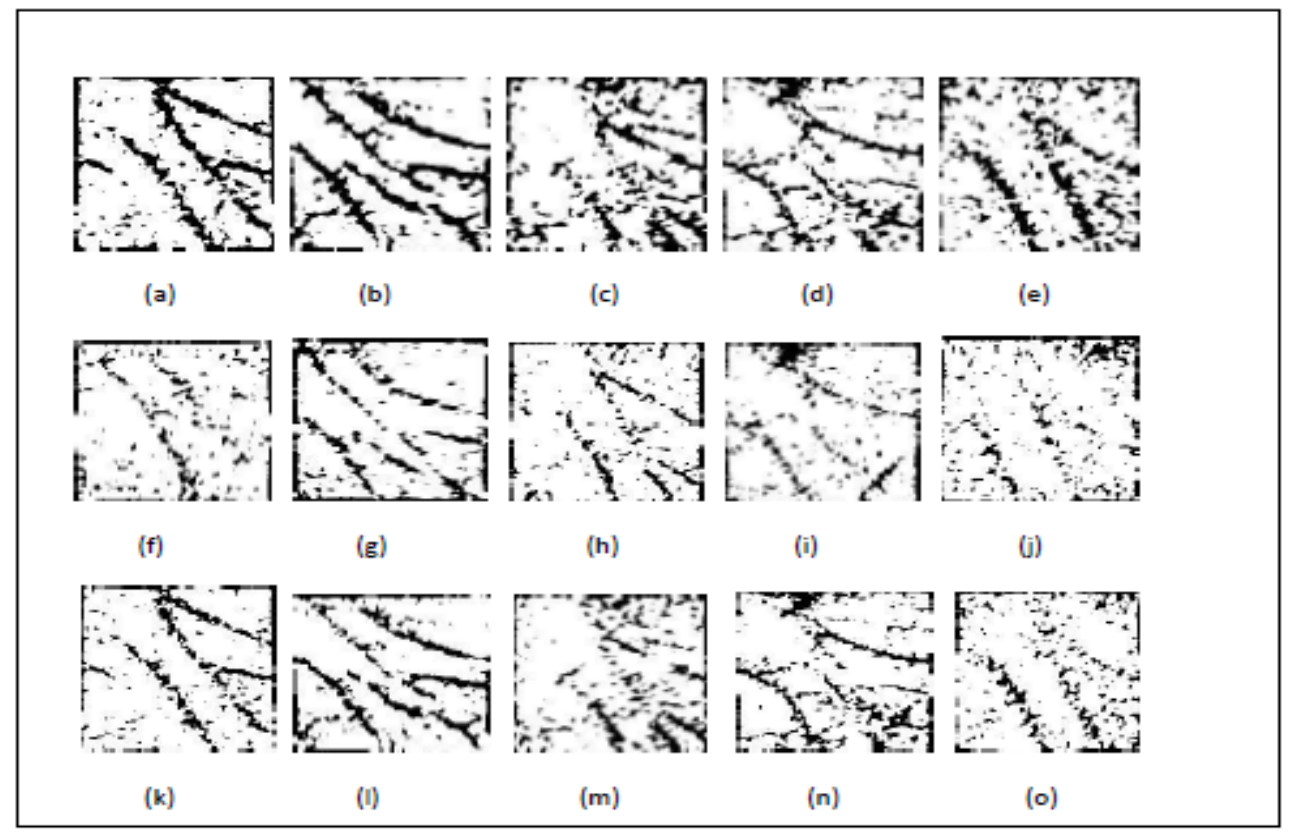

Fig 7: Extracted feature map from (a-e) visible band, (f-j) NIR bands and (k-o) fused images using phase congruency 


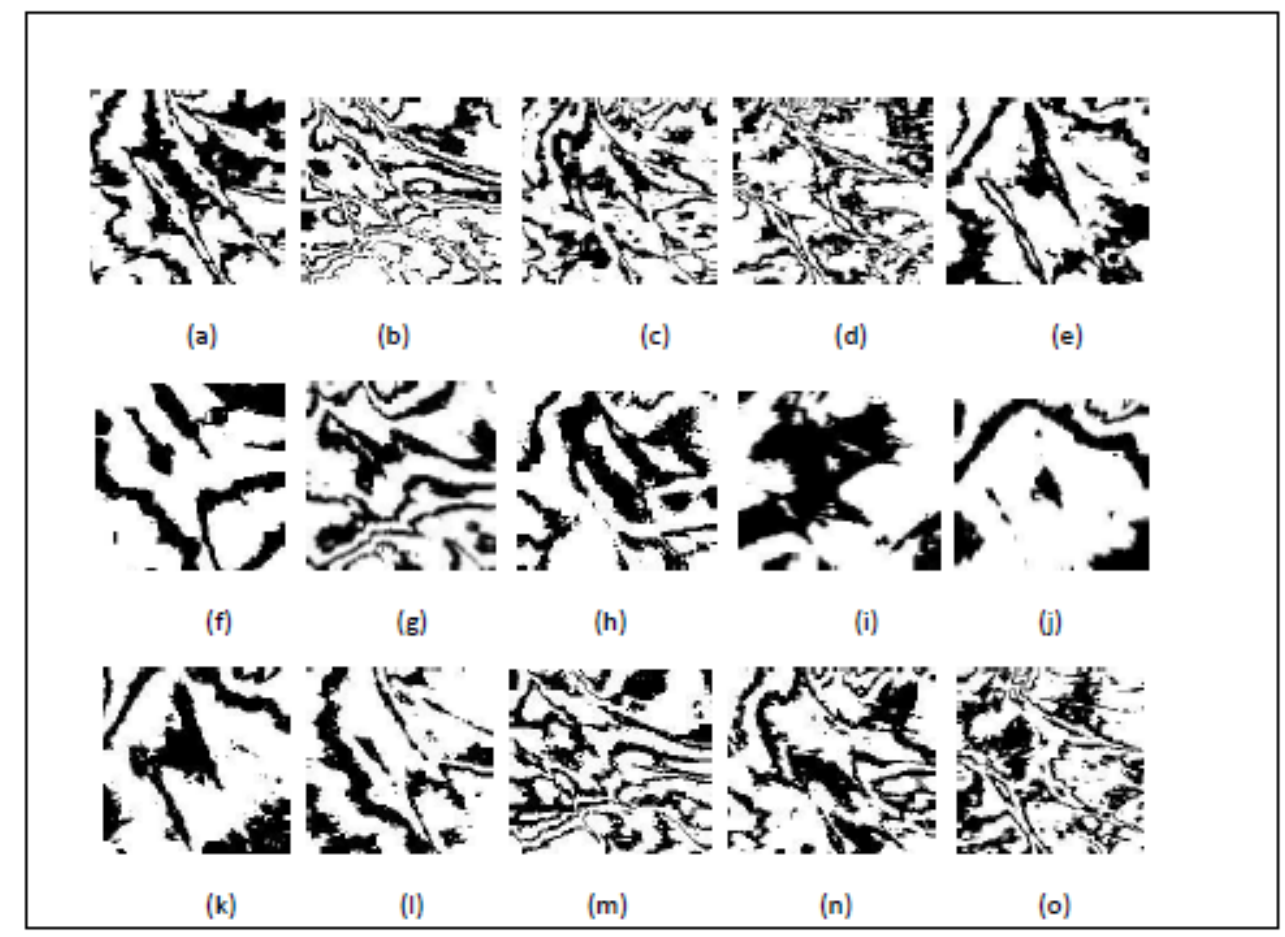

Fig 8: Different Extracted features from (a-e) visible band, (f-j) NIR bands and (k-o) fused images using Gabor filter Table1: Objective evaluation of palm print image features based on matching

\begin{tabular}{|c|c|c|c|c|c|c|c|c|}
\hline \multicolumn{9}{|c|}{ Visible palm print Images } \\
\hline \multirow{2}{*}{$\begin{array}{l}\text { Verification } \\
\text { of Sample } 1 \\
\text { with - }\end{array}$} & \multicolumn{2}{|c|}{ Phase Congruency } & \multicolumn{2}{|c|}{ HoG } & \multicolumn{2}{|c|}{ Adaptive Thresholding } & \multicolumn{2}{|c|}{ Gabor Filter } \\
\hline & $D R D$ & F-Measure & $D R D$ & $\begin{array}{c}F- \\
\text { Measure }\end{array}$ & $D R D$ & $\begin{array}{c}F- \\
\text { Measure }\end{array}$ & $D R D$ & $\begin{array}{c}F- \\
\text { Measure }\end{array}$ \\
\hline Sample 2 & 8.19 & 14.96 & 14.11 & 28.65 & 9.60 & 10.36 & 18.07 & 19.04 \\
\hline Sample 3 & 8.91 & 22.49 & 13.68 & 31.42 & 9.72 & 13.49 & 19.01 & 22.88 \\
\hline Sample 4 & 9.88 & 19.77 & 14.77 & 30.36 & 9.86 & 11.11 & 18.21 & 17.01 \\
\hline Sample 5 & 8.55 & 19.09 & 13.61 & 27.73 & 7.69 & 10.79 & 21.17 & 28.46 \\
\hline Sample 1 & 4.91 & 34.07 & 10.08 & 43.99 & 6.43 & 21.86 & 10.06 & 54.72 \\
\hline \multicolumn{9}{|c|}{ NIR Palm print Images } \\
\hline Sample 2 & 7.03 & 18.89 & 14.48 & 30.43 & 11.88 & 36.32 & 56.48 & 39.81 \\
\hline Sample 3 & 7.43 & 27.63 & 13.88 & 33.33 & 14.88 & 27.75 & 43.79 & 29.42 \\
\hline Sample 4 & 8.70 & 32.08 & 15.75 & 32.73 & 15.96 & 28.31 & 39.25 & 22.79 \\
\hline
\end{tabular}


International Journal of Applied Information Systems (IJAIS) - ISSN : 2249-0868

Foundation of Computer Science FCS, New York, USA

Volume 11 - No. 3, August 2016 - www.ijais.org

\begin{tabular}{|c|c|c|c|c|c|c|c|c|}
\hline Sample 5 & 7.07 & 33.91 & 13.86 & 25.62 & 10.83 & 29.52 & 43.55 & 25.72 \\
\hline Sample 1 & $\mathbf{4 . 5 0}$ & $\mathbf{3 2 . 4 4}$ & $\mathbf{1 1 . 0 9}$ & $\mathbf{4 7 . 8 3}$ & $\mathbf{7 . 6 5}$ & $\mathbf{5 6 . 1 1}$ & $\mathbf{1 1 . 4 9}$ & $\mathbf{7 0 . 3 2}$ \\
\hline
\end{tabular}

Fusion of NIR and Visible Palm Print Images

\begin{tabular}{|c|c|c|c|c|c|c|c|c|}
\hline Sample 2 & 8.23 & 15.81 & 13.33 & 29.3 & 15.44 & 41.57 & 28.95 & 24.25 \\
\hline Sample 3 & 8.235 & 24.69 & 12.49 & 31.15 & 12.72 & 22.42 & 30.09 & 30.23 \\
\hline Sample 4 & 8.93 & 23.00 & 13.61 & 30.48 & 13.21 & 37.96 & 31.27 & 23.60 \\
\hline Sample 5 & 7.72 & 21.91 & 12.28 & 27.24 & 10.35 & 39.71 & 28.57 & 22.55 \\
\hline Sample 1 & $\mathbf{4 . 3 7}$ & $\mathbf{2 8 . 7 8}$ & $\mathbf{1 0 . 2 8}$ & $\mathbf{4 2 . 8 6}$ & $\mathbf{5 . 2 8}$ & $\mathbf{5 9 . 4 3}$ & $\mathbf{1 2 . 7 9}$ & $\mathbf{8 2 . 1 1}$ \\
\hline
\end{tabular}

